\title{
Reducing the Pore Size of Covalent Organic Frameworks in Thin-Film Composite Membranes Enhances Solute Rejection
}

\author{
Amanda R. Corcos, ${ }^{1, \ddagger}$ Gabrielle A. Levato, ${ }^{2, \ddagger}$ Zhiwei Jiang, ${ }^{3}$ Austin M. Evans, ${ }^{1}$ Andrew G. Livingston, ${ }^{3}$ \\ Benito J. Mariñas, ${ }^{2}$ and William R. Dichtel ${ }^{1, *}$ \\ ${ }^{1}$ Department of Chemistry, Northwestern University, 2145 Sheridan Road, Evanston, Illinois 60208, United States \\ ${ }^{2}$ Safe Global Water Institute, Department of Civil and Environmental Engineering, University of Illinois at Urbana-Champaign, Urbana, \\ Illinois 61801, United States \\ ${ }^{3}$ Barrer Centre, Department of Chemical Engineering, Imperial College London, South Kensington Campus, Exhibition Road, London SW7 \\ 2AZ, United Kingdom
}

\begin{abstract}
Three imine-linked covalent organic framework (COF) films are incorporated as active layers into separate thin-film composite (TFC) membranes and tested for their ability to reject an organic pollutant surrogate and salt from water. The synthesized membranes consist of a polyacrylonitrile (PAN) membrane supporting a TAPB-PDA-H, TAPB-PDA-Me, or TAPB-PDA-Et COF thin film. The latter two COFs direct six methyl and ethyl substituents per tiled hexagon into the pores, respectively, while maintaining the same topology across the series. These substituents decrease the effective pore size of the COF compared to the parent TAPB-PDA-H COF. The TAPB-PDA-Me membrane rejects Rhodamine-WT (R-WT) dye and $\mathrm{NaCl}$ better than the TAPB-PDA-H membrane, and the TAPB-PDA-Et membrane exhibits the best rejection overall. The solution-diffusion model used to analyze this permeation behavior indicates that there is a systematic difference in rejection as subsequent pendant groups are added to the interior of the COF pore. These findings demonstrate the concept of tuning the selectivity of COF membranes by systematically reducing the effective pore size within a given topology.
\end{abstract}

Potable water is an increasingly limited resource because of the compounding effects of population growth, industrial pollution and economic development, and in many regions, the effects of climate change. ${ }^{1}$ Inadequate sanitation and the re-use of inadequately treated wastewater represent additional sources of contamination. State-of-the-art pressure-driven membrane purification and desalination systems involve forcing contaminated and brackish water through nanofiltration (NF) and/or reverse osmosis (RO) thin-film composition (TFC) membranes with a thin $(10-250 \mathrm{~nm})$ active layer commonly made of fully- or semi-aromatic polyamide linkages. ${ }^{2}$ These NF and RO systems are more energy efficient than evaporation methods, including multiple effect evaporation, yet still consume energy above the thermodynamic limit. ${ }^{2}$ Polyamide membranes have been mostly developed and optimized empirically, which complicates tuning their separation behavior to specific separation challenges or water matrices. Because their pores cannot be controlled in a designed or atomically precise way, it is difficult to rationally improve their performance since there is a trade-off between energy consumption, flux, salt or pollutant rejection, and other performance characteristics.

Recently, 2D covalent organic frameworks (2D COFs) have emerged as a class of materials with precisely designed porosity, topology, and chemical functionality. ${ }^{3-10}$ These attributes make COFs promising candidates for molecularly engineered membranes since, by careful selection of the monomer subunits which are then polymerized into layered sheets, membranes can be engineered at the
Scheme 1.

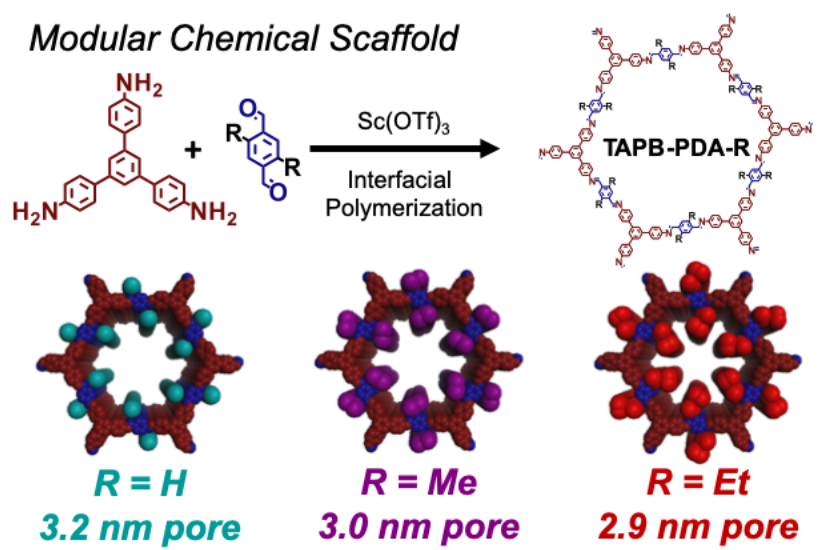

molecular level. To date, hundreds of structurally and chemically unique COFs have been synthesized. However, most COFs have only been synthesized as microcrystalline powders, which bottlenecks their promise for use in membranes. The ability to control material morphology, whether into crystalline films, ${ }^{11-12}$ monolithic blocks, ${ }^{13}$ or colloidal suspensions, ${ }^{14-16}$ represents a major frontier in $2 \mathrm{D}$ polymer science.

Capitalizing on the ability to form crystalline, free-standing COF films, early studies have focused on incorporating these films into membranes and examining their ability to separate trace contaminants from solution, many of which have been described in recent 
reviews. ${ }^{17-19}$ We have shown that the imine-linked TAPB-PDA-H $\mathrm{COF}$ forms as a film via interfacial polymerization at either the liquid/liquid or liquid/air interface when a $\mathrm{Sc}(\mathrm{OTf})_{3}$ catalyst is phase segregated from the TAPB and PDA monomers. ${ }^{20-21}$ The resulting films range in thickness from $100 \mu \mathrm{m}$ down to $2.5 \mathrm{~nm}$ and, when combined with polyethersulfone (PES) supports, the thinnest films of these COFs reject Rhodamine-WT (R-WT) dye and sodium chloride $(\mathrm{NaCl})$ from water over broad pressure ranges. Banerjee and co-workers ${ }^{22}$ synthesized $\beta$-ketoenamine-linked crystalline COF films via interfacial polymerization with a Brønsted acid at a liquid/liquid interface formed by slow diffusion. The films were relatively thick (2.1-5.3 $\mu \mathrm{m})$ with pores around $25 \AA$ and, when they were combined with a polyester non-woven porous support, rejected organic pollutants from water at 0.5 bar upstream pressure. These materials also exhibit high permeance towards organic solvents. ${ }^{23} \mathrm{Lai}^{24-25}$ Meng and Caro, ${ }^{26} \mathrm{Li}$ and $\mathrm{Ma},{ }^{27} \mathrm{Liu}$ and $\mathrm{Wang}{ }^{28}$, and Wei and Wang ${ }^{29}$ have all recently published reports on the ability of COF membranes to reject and separate surrogates of organic pollutants. One of the singular promises of framework-based membranes is the ability to rationally vary the composition and functionality of their pores, yet there are no reports that correlate membrane rejection with systematic structural changes to the COF pore. Herein we report such a study by changing the effective pore size of an iminelinked COF film. These thin, free-standing films are incorporated into the TFC NF membrane structure prior to being tested for their ability to reject an organic pollutant surrogate and salt. The TFC NF membrane, consisting of a TAPB-PDA-H COF film on a polyacrylonitrile (PAN) support, exhibits comparable rejection to our previous studies but is more resistant to degradation in the presence of organic solvents. The modified TAPB-PDA-R COF membranes, which result from systematically decreasing the effective size of the COF pores upon incorporating either methyl or ethyl groups (TAPB-PDA-Me or TAPB-PDA-Et COF, respectively) show enhanced rejection of both $\mathrm{R}-\mathrm{WT}$ and $\mathrm{NaCl}$.

Prior to synthesizing combined COF membranes with different effective pore sizes, microcrystalline COF powders were synthesized from the corresponding monomers and characterized. We recently reported the synthesis of imine-linked COFs, including TAPBPDA-H, using catalytic amounts of the Lewis acid $\mathrm{Sc}(\mathrm{OTf})_{3}$ at room temperature. ${ }^{30}$ Here we used identical reaction conditions to form TAPB-PDA-Me and TAPB-PDA-Et COF powders from 1,3,5tris(4-aminophenyl)benzene (TAPB) and 2,5-disubstituted terephthalaldehyde monomers (PDA-Me or PDA-Et), which were prepared by adapting literature procedures (Scheme 1). ${ }^{31}$ These $\mathrm{COF}$ powders were also synthesized by adapting typical AcOH-catalyzed polymerization conditions at elevated temperature ${ }^{32}$ to ensure that the materials quality was not impeded by the methyl or ethyl substituents on the PDA monomers. Powder X-ray diffraction data (Figures S6-S9) indicate the COF powders formed via either synthetic method are crystalline, as judged by their higher order Bragg diffraction, including 100, 110, 200, 210, 220, 310, and 001 peaks. The average in-plane domain size for both TAPB-PDA-Me and TAPB-PDA-Et is calculated as $35 \mathrm{~nm}$ using the Le Bail Method. Porosimetry measurements for the COF powders synthesized with $\mathrm{Sc}(\mathrm{OTf})_{3}$ (Figures S10-S11) exhibit type IV isotherms that do not completely level off prior to reaching the saturation pressure, and they show slight hysteresis effects upon desorption. COF powders synthesized with $\mathrm{AcOH}$ (S12-S13) exhibit typical type IV isotherms and level off for both TAPB-PDA-Me and TAPB-PDA-Et by 0.6 $P / P_{0}$. Interestingly, TAPB-PDA-Me levels off around $900 \mathrm{~cm}^{3} / \mathrm{g}$ while TAPB-PDA-Et levels off around $870 \mathrm{~cm}^{3} / \mathrm{g}$, consistent with the size of the TAPB-PDA-Et pore being smaller than that of TAPB-PDA-Me. The pore width distribution for the powders formed under room temperature Lewis acid conditions (Figures S14-S15) was slightly larger than those for powders formed under elevated temperature Brønsted acid conditions (Figures S16-S17), but both routes produce materials with a smaller pore size for TAPB-PDA-Et than that for TAPB-PDA-Me. The calculated pore width for TAPB-PDA-H is $3.2 \mathrm{~nm}$, which decreases to $3.0 \mathrm{~nm}$ and then $2.9 \mathrm{~nm}$ for TAPB-PDA-Me and TAPB-PDA-Et, respectively. The measured pore width distribution similarly shows a decrease in size from $3.3 \mathrm{~nm}$ to $3.2 \mathrm{~nm}$ from TAPB-PDA-Me to TAPB-PDAEt. Brunauer-Emmett-Teller (BET) surface areas for TAPB-PDAMe and TAPB-PDA-Et synthesized using either set of reaction conditions were $1800-1900 \mathrm{~m}^{2} / \mathrm{g}$ at relative pressure $\left(P / P_{0}\right)$ between $0.05-0.1$, indicative of high-quality porous crystalline materials. It should be noted that the surface area for TAPB-PDA-Me $(1830$ $\mathrm{m}^{2} / \mathrm{g}$ using $\mathrm{Sc}(\mathrm{OTf})_{3}$ conditions, $1850 \mathrm{~m}^{2} / \mathrm{g}$ using $\mathrm{AcOH}$ conditions $)$ is similar to that of TAPB-PDA-Et $\left(1870 \mathrm{~m}^{2} / \mathrm{g}\right.$ under $\mathrm{Sc}(\mathrm{OTf})_{3}$ conditions, $1900 \mathrm{~m}^{2} / \mathrm{g}$ under $\mathrm{AcOH}$ conditions), which matches the small increase calculated in Connolly surface areas between TAPB-PDA-Me and TAPB-PDA-Et $\left(2450 \mathrm{~m}^{2} / \mathrm{g}\right.$ vs 2500 $\mathrm{m}^{2} / \mathrm{g}$, respectively). Elemental analyses for TAPB-PDA-Me and TAPB-PDA-Et powders formed using $\mathrm{Sc}(\mathrm{OTf})_{3}$ correspond reasonably well to the expected elemental compositions.

Imine-linked COF thin films were subsequently synthesized using an analogous procedure to that used in our previous report. ${ }^{21}$ TAPB and PDA-H, PDA-Me, or PDA-Et were dissolved in separate volumes of 4:1 dioxane:mesitylene, combined into a stock solution, and carefully layered on top of an aqueous solution containing the $\mathrm{Sc}(\mathrm{OTf})_{3}$ catalyst. As seen previously, ${ }^{20-21}$ film thickness could be controlled by altering the concentration or absolute volume of organic material polymerized (see SI). It should be noted that, unlike the combined TAPB-PDA-H stock solution which remained colorless for indefinite periods of time in the absence of catalyst, upon addition of TAPB to PDA-Me or PDA-Et at high concentrations, a yellow precipitate quickly formed even in the absence of the $\mathrm{Sc}(\mathrm{OTf})_{3}$ catalyst. To minimize this background reactivity, solutions of TAPB and PDA-Me or PDA-Et were only combined into a stock solution immediately prior to membrane formation, and if the stock solution turned yellow or formed a precipitate then it was no longer used and a new, fresh stock solution was prepared (see SI).
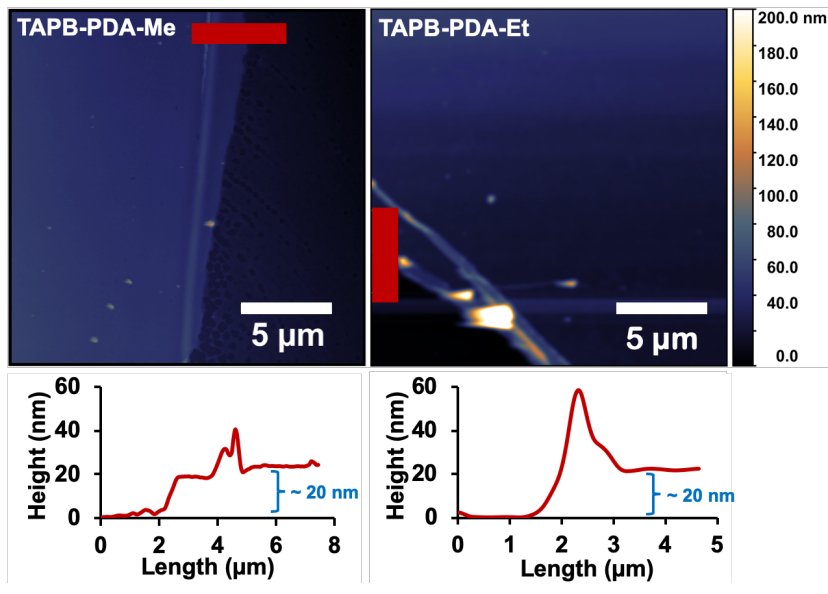

Figure 1. Atomic force microscopy (AFM) topography images of TAPB-PDA-Me film and TAPB-PDA-Et film. AFM height profile for corresponding film included under corresponding micrograph. 

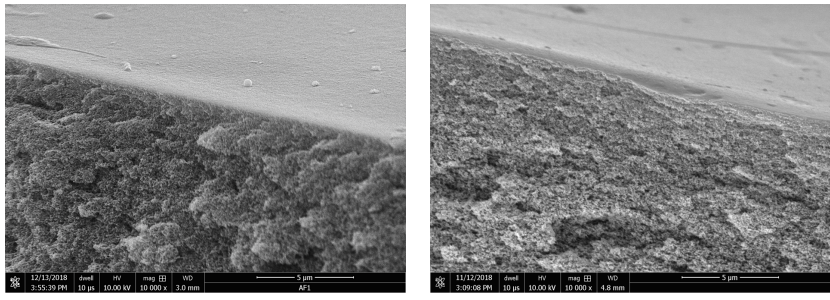

Figure 2. SEM cross-sectional image of thin-film composite PAN membrane containing TAPB-PDA-Me active layers (left). Control image of PAN membrane (right) included for comparison. Images were taken after membranes were exposed to organic solvent; no densification is observed. See Figures S23-30 for additional images.

The interfacially polymerized COF films were characterized by Fourier-transform infrared spectroscopy (FTIR), atomic force microscopy (AFM), optical microscopy, and grazing incidence wide angle X-ray scattering (GI-WAXS), which identified these materials as consistent with the formation of imine-linked networks. As expected of the vibrational data, the amine stretch of TAPB and the aldehyde stretch of PDA-R disappear upon condensation, and a new imine stretch appears for both the COF TAPB-PDA-Me and TAPB-PDA-Et powders and films (Figure S18). The resulting COF films are relatively large in dimension since we can alter the film coverage by changing the size of the polymerization interface (Figure S19). They have a thickness of approximately $20 \mathrm{~nm}$ (Figure 1) and are relatively smooth with a roughness of $3 \mathrm{~nm}$ and $6 \mathrm{~nm}$ for TAPBPDA-Me and TAPB-PDA-Et films, respectively. The comparable film thicknesses indicate that the rates of polymerization were similar, so any differences in solute rejection tests could be associated to differences in COF pore size. We attempted GI-WAXS at the Advanced Photon Source at Argonne National Lab so as to obtain oriented crystallographic information about these films. While we cannot confirm crystallinity for TAPB-PDA-H (Figure S20), TAPBPDA-Me (Figure S21), or TAPB-PDA-Et (Figure S22) films, studies by $\mathrm{us}^{20}$ and others ${ }^{25}$ indicate it is difficult to obtain confirmation of oriented crystallinity of COF films at this thickness. It is possible that we have synthesized a structure that: (a) is completely amorphous, (b) has local order but not long-range order (and/or lacks the regular stacking required for coherent diffraction), or (c) has long-range order but the accompanying diffraction signal is too weak to measure. Our previous studies ${ }^{20-21}$ showed that thick films $(100$ $\mu \mathrm{m})$ of TAPB-PDA-H are crystalline when prepared using this interfacial methodology, but they are too thick for performing membrane experiments. Instead we used thin films, despite their lack of observable diffraction, because they provide less resistance and therefore allow high water flux through the membrane at low energy inputs.

Thin-film composite membranes containing TAPB-PDA-H, TAPB-PDA-Me, or TAPB-PDA-Et active layers were synthesized and analyzed using scanning electron microscopy (SEM). A similar procedure was used as described previously, ${ }^{20}$ in which free-standing imine-linked COF films were fabricated and transferred onto polyacrylonitrile (PAN) using Langmuir-Shaeffer techniques. The PAN was synthesized according to literature procedure ${ }^{33}$ and chosen as the membrane support since it is resistant to decomposition in organic solvent. Previous work with TAPB-PDA-H thin-film COF membranes on a polyethersulfone (PES) support highlighted the incompatibility between the 1,4-dioxane:mesitylene solvent mixture required for the COF film synthesis and the PES material. ${ }^{20} \mathrm{SEM}$ images of the combined COF and PES membranes revealed the occurrence of PES densification after solvent exposure, which appeared to decrease the water permeability without affecting solute rejection of the combined membranes. The PAN used in this work did not undergo any appreciable densification upon solvent exposure, as seen in multiple SEM images (see Figures 2, S23-25 for cross-sectional images, and Figures S26-S30 for top-down images), and therefore was considered as a superior alternative.

TFC membranes of TAPB-PDA-H, TAPB-PDA-Me, or TAPBPDA-Et on PAN were each tested for their water permeability and their ability to reject R-WT and $\mathrm{NaCl}$, which are solutes used as surrogates for organic contaminants and background electrolytes, respectively. The solute rejection results are shown in Figure 3 with

corresponding permeate fluxes shown in Figures 4 and S31-S32. These TFC membranes exhibit lower water permeability but enhanced $\mathrm{R}-\mathrm{WT}$ and $\mathrm{NaCl}$ rejection when compared to the unmodified PAN support. We compared the performance of the TFC COF membranes to those of each PAN support before (pristine) and after (control) solvent exposure, as well as to the commercial nanofiltration membrane DOW FILMTEC NF-270. Solvent exposure did not affect membrane support performance, indicating that the COF film
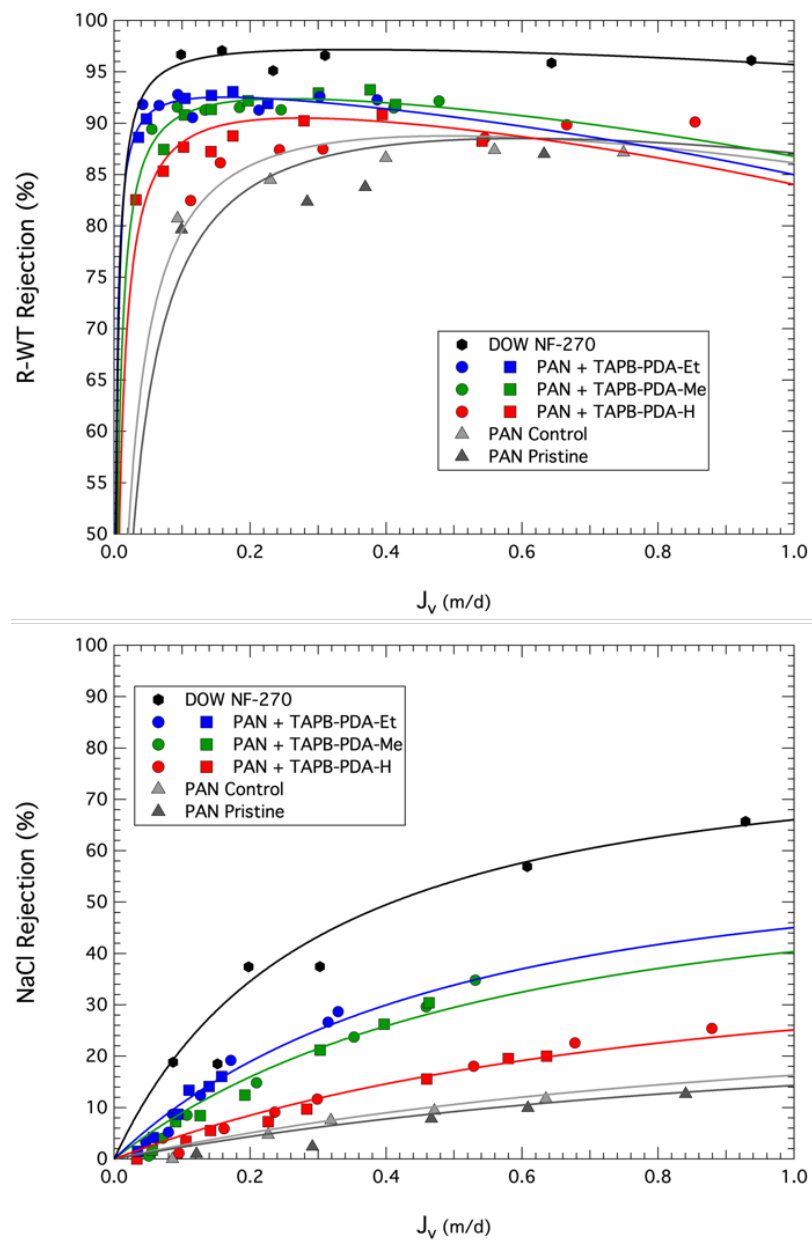

Figure 3. Rejection of surrogate organic contaminant Rhodamine-WT (R-WT) (upper plot) and representative electrolyte $\mathrm{NaCl}$ (lower plot) as a function of permeate flux $\mathrm{J}_{\mathrm{v}}$ obtained for thin-film composite (TFC) polyacrylonitrile (PAN) membranes with COF active layers of TAPBPDA-H, TAPB-PDA-Me, or TAPB-PDA-Et. Data also shown for corresponding pristine and control PAN supports without the COF active layer, and for commercial nanofiltration (NF) membrane (DOW FILMTEC NF-270). 


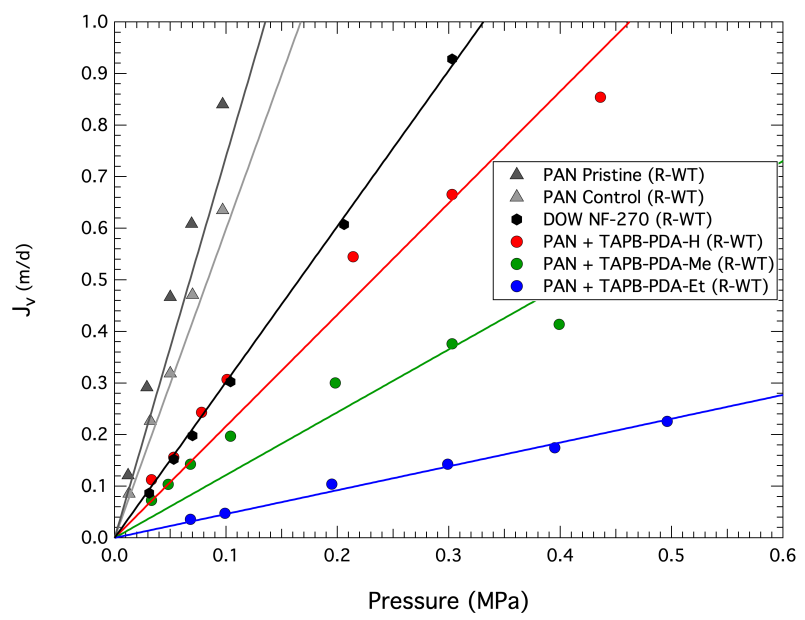

Figure 4. Permeate water flux $\mathrm{J}_{\mathrm{v}}$ as a function of hydraulic pressure obtained for thin-film composite polyacrylonitrile (PAN) membranes with COF active layers of TAPB-PDA-H, TAPB-PDA-Me, or TAPB-PDAEt tested with R-WT solutions (see Figure S31 for those tested with $\mathrm{NaCl}$ solutions and Figure $\mathrm{S} 32$ for combined regression lines). Data and regression lines are also shown for corresponding pristine and control PAN supports without the COF layer, and for commercial nanofiltration (NF) membrane (DOW FILMTEC NF-270).

itself plays an integral part in enhanced solute rejection. For example, at a low permeate water flux of $0.02 \mathrm{~m} / \mathrm{d}, 49.0 \%$ of R-WT is rejected by the (control) PAN support, and rejection increases to $71.0 \%, 77.6 \%$, or $86.2 \%$ upon addition of the TAPB-PDA-H, TAPB-PDA-Me, or TAPB-PDA-Et COF film, respectively. When the permeate water flux is increased to $0.1 \mathrm{~m} / \mathrm{d}$, R-WT rejection increases from $79.6 \%$ for the (control) PAN up to $88.1 \%, 90.8 \%$, or 92.3\% depending on the COF thin film added (TAPB-PDA-H, TAPB-PDA-Me, or TAPB-PDA-Et, respectively). It is critical to point out that increasing rejection at low pressure is very difficult since the diffusive transport of solutes through the membrane pores is more prevalent than at higher pressure for which higher advective transport takes place, and so these data best exhibit the differences between membrane materials. The R-WT rejection data shows hallmarks of separations associated with well-defined materials since there is increased rejection by the TFC COF membranes containing TAPB-PDA-H versus TAPB-PDA-Me versus TAPB-PDA-Et active layers. This trend is also seen in the $\mathrm{NaCl}$ rejection data. At a permeate water flux of $0.1 \mathrm{~m} / \mathrm{d}, 2.71 \%$ of $\mathrm{NaCl}$ is rejected by the (control) PAN support, and rejection increased to $4.61 \%, 9.03 \%$, or $10.9 \%$ upon addition of the TAPB-PDA-H, TAPB-PDA-Me, or TAPB-PDA-Et COF film, respectively. Even at a higher permeate water flux of $0.5 \mathrm{~m} / \mathrm{d}, \mathrm{NaCl}$ rejection increases from $10.6 \%$ for the (control) PAN support, up to $17.1 \%, 29.5 \%$, or $33.9 \%$ upon addition of the TAPB-PDA-H, TAPB-PDA-Me, or TAPB-PDA-Et COF film, respectively. Thus, all of the COF membranes provide measurable, enhanced rejection of both $\mathrm{R}-\mathrm{WT}$ and $\mathrm{NaCl}$ compared to the (control) PAN support and, furthermore, the data revealed a noticeable trend related to the type of TAPB-PDA-R used in the active layer: as the pendant group size increases from a hydrogen atom in TAPB-PDA-H to a methyl group in TAPB-PDA-Me and finally to an ethyl group in TAPB-PDA-Et, there is a systematic increase in rejection of both R-WT and $\mathrm{NaCl}$.

The rejection data shown in Figures 3 and 4 were modeled using solution-diffusion model equations ${ }^{20}$ and fit the experimental data points well. One of two equations were used to fit the data depending on whether a thin-film COF active layer was included in the membrane. The solute rejection data sets collected for pristine and control PAN shown in Figure 3 were fitted with the solution diffusion model (Eq. S1), which accounted for: solute diffusive permeation $(B[\mathrm{~m} / \mathrm{d}])$, fraction of water flux passing through active layer imperfections ( $\alpha$ [dimensionless]), solution mass transfer coefficient in concentration-polarization film $(\mathrm{k}[\mathrm{m} / \mathrm{d}])$, and overall permeate flux $\left(\mathrm{J}_{\mathrm{v}}[\mathrm{m} / \mathrm{d}]\right)$. Rejection data for both R-WT and $\mathrm{NaCl}$ were experimentally tested in duplicate for each of the TFC membranes containing a COF active layer (TAPB-PDA-H, TAPB-PDA-Me, and TAPB-PDA-Et), and they were combined and fitted with a modified two-film solution diffusion model (Eq. S2) to determine the intrinsic properties of the $\mathrm{COF}$ active layers separate from the PAN support. ${ }^{20}$ The resulting fitted lines are depicted in Figure 3 and the corresponding fitting parameters are listed in Table 1 , along with the water permeation coefficients $(\mathrm{A}[\mathrm{m} /(\mathrm{d} \times \mathrm{MPa})])$ obtained

Table 1. Water and solute transport parameters obtained by fitting the experimental data in Figures 3 and 4 . Parameters B and $\alpha$ for R-WT and $\mathrm{NaCl}$ listed for the $\mathrm{COF}$ membranes are intrinsic transport parameters for the COF active layer obtained by fitting the corresponding data in Figure 3 with Equation S2 using the parameters obtained by fitting the solute rejection data for the control PAN support in Figure 3 with Equation $\mathrm{S} 1$. To observe comparable changes in $\mathrm{B}$ values for $\mathrm{NaCl}$, the R-WT a value was used in $\mathrm{NaCl}$ modeling.

\begin{tabular}{|c|c|c|c|c|c|c|}
\hline \multirow{2}{*}{ Membrane } & \multicolumn{3}{|c|}{ R-WT } & \multicolumn{3}{|c|}{$\mathrm{NaCl}$} \\
\hline & $\mathbf{A}\left(\mathbf{m} / \mathbf{d}^{*} \mathbf{M P a}\right)$ & B $(\mathbf{m} / \mathbf{d})$ & $\boldsymbol{\alpha}(-)$ & $\mathbf{A}\left(\mathbf{m} /\left(\mathbf{d}^{*} \mathrm{MPa}\right)\right.$ & $\mathbf{B}(\mathbf{m} / \mathbf{d})$ & $\boldsymbol{\alpha}(-)$ \\
\hline Pristine PAN & $5.59 \pm 0.18$ & $0.026 \pm 0.006$ & $0.022 \pm 0.011$ & $7.39 \pm 0.25$ & $3.96 \pm 0.28$ & 0.022 \\
\hline Control PAN & $6.39 \pm 0.30$ & $0.019 \pm 0.003$ & $0.032 \pm 0.008$ & $5.99 \pm 0.19$ & $3.35 \pm 0.22$ & 0.032 \\
\hline PAN + TAPB-PDA-H & $2.16 \pm 0.13$ & $0.009 \pm 0.002$ & $0.065 \pm 0.008$ & $2.32 \pm 0.10$ & $3.66 \pm 0.25$ & 0.065 \\
\hline PAN + TAPB-PDA-Me & $1.25 \pm 0.06$ & $0.006 \pm 0.001$ & $0.048 \pm 0.004$ & $1.47 \pm 0.07$ & $1.21 \pm 0.08$ & 0.048 \\
\hline PAN + TAPB-PDA-Et & $0.91 \pm 0.04$ & $0.0021 \pm 0.0004$ & $0.055 \pm 0.004$ & $1.2 \pm 0.4$ & $0.92 \pm 0.06$ & 0.055 \\
\hline NF-270 & $3.06 \pm 0.02$ & $0.003 \pm 0.001$ & $0.012 \pm 0.003$ & $3.02 \pm 0.01$ & $0.35 \pm 0.08$ & 0.012 \\
\hline
\end{tabular}


from the slopes of the plots in Figure 4. The mass transfer coefficients used in Equations $\mathrm{S} 1$ and $\mathrm{S} 2$ to represent concentration polarization of R-WT $\left(\mathrm{k}_{\mathrm{R}-\mathrm{WT}}=0.9 \mathrm{~m} / \mathrm{d}\right)$ and $\mathrm{NaCl}\left(\mathrm{k}_{\mathrm{NaCl}}=2.6 \mathrm{~m} / \mathrm{d}\right)$ were obtained from previous analysis with these composite membranes. ${ }^{20}$

Additionally, there is a general decrease in permeate flux as the effective COF pore size decreases with increasing pendant group size. As the pendant groups grow in length, the overall hydrophobicity of the COF film also increases. A more hydrophobic membrane, together with a narrower pore, is consistent with reduced water permeability of the TFC COF membrane. We identified the same decrease in permeability with increasing TAPB-PDA-H COF thickness, ${ }^{20}$ but we also observed no significant change in rejection capabilities. Greater pore constriction and increased hydrophobicity are consistent with the decreased TFC COF membrane permeate flux observed between the three membranes investigated here.

There is also a significant shift in the solute permeation coefficient (B) for the different TFC COF membranes (Table 1), which is consistent with the $\mathrm{COF}$ films presenting greater resistance to diffusive transport with an increasing molecular size of the pendant groups in the TAPB-PDA-R active layers. The change in the B parameter is likely due to increased tortuosity created by the pendant groups. The portion of solute transported through the COF active layer by pore surface diffusion is affected by the molecular size of the pendant groups branching into the COF pores. The pendant groups create obstacles, which slow down the surface diffusion capability and increase the rejection of the solute. In contrast, corresponding changes in the advective transport parameter, $\alpha$, are small, supporting the slight change the TAPB-PDA-R pendant groups have on the COF pore size.

It is also important to note the comparison between the TFC TAPB-PDA-H PAN membranes investigated here and the TFC TAPB-PDA-H PES membrane evaluated previously. ${ }^{20-21}$ Although two different support materials were used, the two-film solution diffusion model (Eq. S2) provided values that are statistically similar when evaluated using a t-test (two-tail distribution, equal variance) for the R-WT data sets $(B=0.009 \pm 0.002$ in the present study and $\mathrm{B}=0.012 \pm 0.002 \mathrm{~m} / \mathrm{d}$ in the previous study $\left.{ }^{20}\right)$. This confirms that the TAPB-PDA-H active layer formed in both studies on different supports are similar. Unfortunately, a direct comparison between the $\mathrm{NaCl}$ data sets was more difficult because the low rejection observed for $\mathrm{NaCl}$ does not allow for accurate differentiation between diffusive and advective transport. As Figures 3 and 4 show, the permeate flux and solute rejection by all COF membranes were lower than those observed for the NF-270 commercial membrane. Decreasing the COF pore size and using supports with higher permeate flux should help to address these differences.

In conclusion, we have developed an interfacial polymerization method to probe thin layers of COF films or related cross-linked networks and incorporated them into TFC membranes with a PAN support. Although these materials do not show evidence of longrange order, their rejection behavior exhibits characteristics associated with a progressive decrease in pore size as the pendant group length in the COF active layer is increased from hydrogen to methyl to ethyl. There is also a significant shift in the solute permeation coefficient, $\mathrm{B}$, consistent with the $\mathrm{COF}$ films presenting greater resistance to diffusive transport with decreasing COF pore size and increasing pendant group length. These results indicate that rational changes can be correlated to membrane performance, even in materials with no observable long-range order. As methods to access extremely thin COF films with unambiguous long-range order (perhaps even as single or few-layer structures) are developed, these results represent an important benchmark towards the importance of structural design and membrane performance.

\section{ASSOCIATED CONTENT}

\section{Supporting Information}

The Supporting Information is available free of charge on the ChemRxiv website:

Materials and instrumentation; experimental procedures and characterizations; ${ }^{1} \mathrm{H}$ and ${ }^{13} \mathrm{C}$ NMR spectra of $\mathrm{COF}$ monomers; powder X-ray diffraction patterns of COF powders; nitrogen adsorption isotherms and BET plots for COF powders; pore distributions for COF powders; vibrational data for $\mathrm{COF}$ monomers, powders, and films; optical microscopy images of $\mathrm{COF}$ films; grazing incidence wide angle $\mathrm{X}$-ray scattering (GI-WAXS) patterns of COF films; SEM images of TFC COF PAN membranes; solution-diffusion model equations; and permeate water flux as a function of hydraulic pressure (PDF).

\section{AUTHOR INFORMATION}

\section{Corresponding Author}

*WRD: wdichtel@northwestern.edu

\section{Author Contributions}

†A.R.C. and G.A.L. contributed equally.

\section{Notes}

The authors declare no competing financial interests.

\section{ACKNOWLEDGMENT}

This research was supported by the National Science Foundation (CBET-1706219) and used resources at the Advanced Photon Source (Sector 8), which is a Department of Energy Office of Science User Facility (DE-AC02-05CH11231). A.M.E. is supported by an NSF Graduate Research Fellowship (DGE-1324585). The UK based team was supported by the Engineering and Physical Sciences Research Council (CBET-EPSRC EP/R018847).

\section{REFERENCES}

1. The United Nations World Water Development Report 2019: Leaving No One Behind; UNESCO: 2019.

2. Elimelech, M.; Phillip, W. A., The Future of Seawater Desalination: Energy, Technology, and the Environment. Science 2011, 333 (6043), 712.

3. Colson, J. W.; Dichtel, W. R., Rationally synthesized two-dimensional polymers. Nat. Chem. 2013, 5 (6), 453-65.

4. DeBlase, C. R.; Dichtel, W. R., Moving Beyond Boron: The Emergence of New Linkage Chemistries in Covalent Organic Frameworks. Macromolecules 2016, 49 (15), 5297-5305.

5. Bisbey, R. P.; Dichtel, W. R., Covalent Organic Frameworks as a Platform for Multidimensional Polymerization. ACS Cent. Sci. 2017, 3 (6), 533 543.

6. Diercks, C. S.; Yaghi, O. M., The atom, the molecule, and the covalent organic framework. Science 2017, 355 (6328), 923.

7. Jin, Y.; Hu, Y.; Zhang, W., Tessellated multiporous two-dimensional covalent organic frameworks. Nat. Rev. Chem. 2017, 1, 0056.

8. Lohse, M. S.; Bein, T., Covalent Organic Frameworks: Structures, Synthesis, and Applications. Adv. Funct. Mater. 2018, 28 (33), 1705553.

9. Kandambeth, S.; Dey, K.; Banerjee, R., Covalent Organic Frameworks: Chemistry beyond the Structure. J. Am. Chem. Soc. 2019, 141 (5), $1807-$ 1822. 
10. Song, Y.; Sun, Q.; Aguila, B.; Ma, S., Opportunities of Covalent Organic Frameworks for Advanced Applications. Adv. Sci. 2019, 6 (2), 1801410.

11. Colson, J. W.; Woll, A. R.; Mukherjee, A.; Levendorf, M. P.; Spitler, E. L.; Shields, V. B.; Spencer, M. G.; Park, J.; Dichtel, W. R., Oriented 2D covalent organic framework thin films on single-layer graphene. Science 2011, 332 (6026), 228-31.

12. Kim, S.; Lim, H.; Lee, J.; Choi, H. C., Synthesis of a Scalable TwoDimensional Covalent Organic Framework by the Photon-Assisted Imine Condensation Reaction on the Water Surface. Langmuir 2018, 34 (30), 8731-8738.

13. Karak, S.; Kandambeth, S.; Biswal, B. P.; Sasmal, H. S.; Kumar, S.; Pachfule, P.; Banerjee, R., Constructing Ultraporous Covalent Organic Frameworks in Seconds via an Organic Terracotta Process. J. Am. Chem. Soc. 2017, 139 (5), 1856-1862.

14. Smith, B. J.; Parent, L. R.; Overholts, A. C.; Beaucage, P. A.; Bisbey, R. P.; Chavez, A. D.; Hwang, N.; Park, C.; Evans, A. M.; Gianneschi, N. C.; Dichtel, W. R., Colloidal Covalent Organic Frameworks. ACS Cent. Sci. 2017, 3 (1), 58-65.

15. Evans, A. M.; Parent, L. R.; Flanders, N. C.; Bisbey, R. P.; Vitaku, E.; Chen, L. X.; Gianneschi, N. C.; Dichtel, W. R., Seeded growth of single-crystal two-dimensional covalent organic frameworks. Science 2018, 361 (6397), 52-57.

16. Li, R. L.; Flanders, N. C.; Evans, A. M.; Ji, W.; Castano, I.; Chen, L. X.; Gianneschi, N. C.; Dichtel, W. R., Controlled growth of imine-linked twodimensional covalent organic framework nanoparticles. Chem. Sci. 2019, 10 (13), 3796-3801.

17. Fernandes, S. P. S.; Romero, V.; Espiña, B.; Salonen, L. M., Tailoring Covalent Organic Frameworks to Capture Water Contaminants. Chem. Eur. J. 2019, 25 (26), 6461-6473.

18. Wang, H.; Zeng, Z.; Xu, P.; Li, L.; Zeng, G.; Xiao, R.; Tang, Z.; Huang, D.; Tang, L.; Lai, C.; Jiang, D.; Liu, Y.; Yi, H.; Qin, L.; Ye, S.; Ren, X.; Tang, W., Recent progress in covalent organic framework thin films: fabrications, applications and perspectives. Chem. Soc. Rev. 2019, 48 (2), 488-516.

19. Yuan, S.; Li, X.; Zhu, J.; Zhang, G.; Van Puyvelde, P.; Van der Bruggen, B., Covalent Organic Frameworks for Membrane Separation. Chem. Soc. Rev. 2019.

20. Valentino, L.; Matsumoto, M.; Dichtel, W. R.; Marinas, B. J., Development and Performance Characterization of a Polyimine Covalent Organic Framework Thin-Film Composite Nanofiltration Membrane. Environ. Sci. Technol. 2017, 51 (24), 14352-14359.

21. Matsumoto, M.; Valentino, L.; Stiehl, G. M.; Balch, H. B.; Corcos, A. R.; Wang, F.; Ralph, D. C.; Marinas, B. J.; Dichtel, W. R., Lewis-Acid-Catalyzed Interfacial Polymerization of Covalent Organic Framework Films. Chem 2018, 4 (2), 308-317.
22. Dey, K.; Pal, M.; Rout, K. C.; Kunjattu, H. S.; Das, A.; Mukherjee, R.; Kharul, U. K.; Banerjee, R., Selective Molecular Separation by Interfacially Crystallized Covalent Organic Framework Thin Films. J. Am. Chem. Soc. 2017, 139 (37), 13083-13091.

23. Kandambeth, S.; Biswal, B. P.; Chaudhari, H. D.; Rout, K. C.; Kunjattu, H. S.; Mitra, S.; Karak, S.; Das, A.; Mukherjee, R.; Kharul, U. K.; Banerjee, R., Selective Molecular Sieving in Self-Standing Porous CovalentOrganic-Framework Membranes. Adv. Mater. 2017, 29 (2), 1603945

24. Gadwal, I.; Sheng, G.; Thankamony, R. L.; Liu, Y.; Li, H.; Lai, Z., Synthesis of Sub-10 nm Two-Dimensional Covalent Organic Thin Film with Sharp Molecular Sieving Nanofiltration. ACS Appl. Mater. Interfaces. 2018, 10 (15), 12295-12299.

25. Shinde, D. B.; Sheng, G.; Li, X.; Ostwal, M.; Emwas, A. H.; Huang, K. W.; Lai, Z., Crystalline 2D Covalent Organic Framework Membranes for High-Flux Organic Solvent Nanofiltration. J. Am. Chem. Soc. 2018, 140 (43), 14342-14349.

26. Fan, H.; Gu, J.; Meng, H.; Knebel, A.; Caro, J., High-Flux Membranes Based on the Covalent Organic Framework COF-LZU1 for Selective Dye Separation by Nanofiltration. Angew. Chem., Int. Ed. 2018, 57 (15), 4083 4087.

27. Zhang, W.; Zhang, L.; Zhao, H.; Li, B.; Ma, H., A two-dimensional cationic covalent organic framework membrane for selective molecular sieving. J. Mater. Chem. A 2018, 6 (27), 13331-13339.

28. Hao, Q.; Zhao, C.; Sun, B.; Lu, C.; Liu, J.; Liu, M.; Wan, L.-J.; Wang, D., Confined Synthesis of Two-Dimensional Covalent Organic Framework Thin Films within Superspreading Water Layer. J. Am. Chem. Soc. 2018, 140 (38), 12152-12158.

29. Zhou, W.; Wei, M.; Zhang, X.; Xu, F.; Wang, Y., Fast Desalination by Multilayered Covalent Organic Framework (COF) Nanosheets. ACS Appl. Mater. Interfaces 2019, 11 (18), 16847-16854.

30. Matsumoto, M.; Dasari, R. R.; Ji, W.; Feriante, C. H.; Parker, T. C.; Marder, S. R.; Dichtel, W. R., Rapid, Low Temperature Formation of ImineLinked Covalent Organic Frameworks Catalyzed by Metal Triflates. J. Am. Chem. Soc. 2017, 139 (14), 4999-5002.

31. Wessig, P.; Gerngroß, M.; Freyse, D.; Bruhns, P.; Przezdziak, M.; Schilde, U.; Kelling, A., Molecular Rods Based on Oligo-spiro-thioketals. J. Org. Chem. 2016, 81 (3), 1125-1136.

32. Smith, B. J.; Overholts, A. C.; Hwang, N.; Dichtel, W. R., Insight into the crystallization of amorphous imine-linked polymer networks to $2 \mathrm{D}$ covalent organic frameworks. Chem. Commun. 2016, 52 (18), 3690-3693.

33. Cook, M.; Gaffney, P. R. J.; Peeva, L. G.; Livingston, A. G., Roll-toroll dip coating of three different PIMs for Organic Solvent Nanofiltration. J. Membrane Sci. 2018, 558, 52-63. 
Table of Contents Image

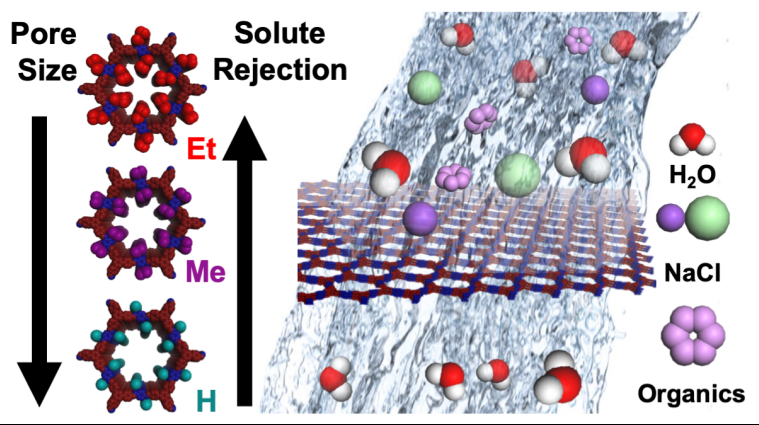

